\title{
TRACE THEOREMS: CRITICAL CASES AND BEST CONSTANTS
}

\author{
MICHAEL RUZHANSKY AND MITSURU SUGIMOTO
}

\begin{abstract}
The purpose of this paper is to present the critical cases of the trace theorems for the restriction of functions to closed surfaces, and to give the asymptotics for the norms of the traces under dilations of the surface. We also discuss the best constants for them.
\end{abstract}

\section{INTRODUCTION}

It is very well known that if $s>1 / 2$ and $\Sigma \subset \mathbb{R}^{n}$ is a closed hypersurface, $n \geq 2$, then we have the following trace theorem:

$$
\left\|f_{\mid \Sigma}\right\|_{L^{2}(\Sigma ; d \omega)} \leq C\|f\|_{H^{s}\left(\mathbb{R}^{n}\right)},
$$

where $H^{s}$ is the Sobolev space over $L^{2}$ and $d \omega$ is the induced surface measure on $\Sigma$. It is also known that (1.1) fails for $s=1 / 2$. The purpose of this note is to show how (1.1) can be modified to still hold for $s=1 / 2$. Moreover, if the origin belongs to the set bounded by $\Sigma$, we investigate the dependence of the constant $C$ in (1.1) on a parameter $\rho \rightarrow \infty$ when we replace $(\Sigma ; d \omega)$ by its dilation $\left(\rho \Sigma ; \rho^{n-1} d \omega\right)$. We also find best constants for some instances of (1.1).

We give a simple proof of such results by deriving estimates for different traces from the global smoothing estimates for dispersive equations that have been established by the authors in [15], [16] by the geometric analysis using the methods of canonical transforms and comparison principles developed for the smoothing estimates. We note that usually the argument is converse and one derives both the smoothing estimates and the limiting absorption principle from the appropriate trace theorem (see e.g. Ben-Artzi and Klainerman [4). However, in this instance, we show how new arguments and methods in PDEs can be applied to deduce facts about traces.

Let us formulate our results. Let $a \in C^{\infty}\left(\mathbb{R}^{n} \backslash 0\right)$ be real-valued and satisfy $a(\xi)>$ 0 for all $\xi$. We will be restricting to the level set of the function $a$ defined by $\Sigma_{a}=\left\{\xi \in \mathbb{R}^{n} \backslash 0: a(\xi)=1\right\}$. In order to simplify the exposition, let us modify the function $a$ outside the set $\Sigma_{a}$ so that $a$ becomes positively homogeneous of order two, namely, we can assume that $a$ is already positively homogeneous satisfying $a(\lambda \xi)=\lambda^{2} a(\xi)$ for $\lambda>0$ and $\xi \neq 0$. The dual hypersurface $\Sigma_{a}^{*}$ is defined by $\Sigma_{a}^{*}=\left\{\nabla a(\xi): \xi \in \Sigma_{a}\right\}$, and the dual function $a^{*}(\xi)$ can be determined by the relation $\Sigma_{a^{*}}=\Sigma_{a}^{*}$. We discuss some of its properties in Section 2,

As usual, we denote by $H^{s}\left(\mathbb{R}^{n}\right)$ and $\dot{H}^{s}\left(\mathbb{R}^{n}\right)$ the Sobolev spaces with the norms $\|g\|_{H^{s}\left(\mathbb{R}^{n}\right)}=\left\|\left\langle D_{x}\right\rangle^{s} g\right\|_{L^{2}\left(\mathbb{R}^{n}\right)}$ and $\|g\|_{\dot{H}^{s}\left(\mathbb{R}^{n}\right)}=\left\|\left|D_{x}\right|^{s} g\right\|_{L^{2}\left(\mathbb{R}^{n}\right)}$, respectively. We use the notation $D_{x}=\frac{1}{i} \nabla_{x}$, so that $\left|D_{x}\right|=\sqrt{-\Delta},\left\langle D_{x}\right\rangle=\sqrt{1-\Delta}$, and for a function $c(\xi)$

Date: November 11, 2018.

The first author was supported by the EPSRC Leadership Fellowship EP/G007233/1. 
we denote by $c\left(D_{x}\right)$ the Fourier multiplier $c\left(D_{x}\right)=\mathcal{F}^{-1} c(\xi) \mathcal{F}$, where $\mathcal{F}$ denotes the Fourier transformation and $\mathcal{F}^{-1}$ its inverse defined by

$$
\mathcal{F} f(\xi)=\widehat{f}(\xi)=\int_{\mathbb{R}^{n}} e^{-i x \cdot \xi} f(x) d x, \quad \mathcal{F}^{-1} f(x)=\widehat{f}^{*}(x)=\frac{1}{(2 \pi)^{n}} \int_{\mathbb{R}^{n}} e^{i x \cdot \xi} f(\xi) d \xi .
$$

Theorem 1.1. Let $a(\xi) \in C^{\infty}\left(\mathbb{R}^{n} \backslash 0\right)$ be real-valued and satisfy $a(\xi)>0$ and $a(\lambda \xi)=$ $\lambda^{2} a(\xi)$ for $\lambda>0$ and $\xi \neq 0$. Let $\Sigma_{a}=\left\{\xi \in \mathbb{R}^{n} \backslash 0: a(\xi)=1\right\}$. Suppose $s>1 / 2$. Then we have

$$
\left\|f_{\mid \Sigma_{a}}\right\|_{L^{2}\left(\Sigma_{a} ; d \omega\right)} \leq C\|f\|_{H^{s}\left(\mathbb{R}^{n}\right)} .
$$

Moreover, for $1 / 2<s<n / 2$, we have

$$
\left\|f_{\mid \Sigma_{a}}\right\|_{L^{2}\left(\Sigma_{a} ; d \omega\right)} \leq C\|f\|_{\dot{H}^{s}\left(\mathbb{R}^{n}\right)} .
$$

If we in addition assume that the Gaussian curvature of $\Sigma_{a}$ is non-vanishing, then we have also the critical cases

$$
\left\|\left(\frac{\nabla a(x)}{|\nabla a(x)|} \wedge \frac{D_{x}}{\left|D_{x}\right|}\right) f_{\mid \Sigma_{a}}\right\|_{L^{2}\left(\Sigma_{a} ; d \omega\right)} \leq C\|f\|_{\dot{H}^{1 / 2}\left(\mathbb{R}^{n}\right)}
$$

and

$$
\left\|\left(\frac{x}{|x|} \wedge \frac{\nabla a^{*}\left(D_{x}\right)}{\left|\nabla a^{*}\left(D_{x}\right)\right|}\right) f_{\mid \Sigma_{a}}\right\|_{L^{2}\left(\Sigma_{a} ; d \omega\right)} \leq C\|f\|_{\dot{H}^{1 / 2}\left(\mathbb{R}^{n}\right)},
$$

where $a^{*}(x)$ is the dual function of $a(\xi)$.

Here the outer product $p \wedge q$ of vectors $p=\left(p_{1}, p_{2}, \ldots, p_{n}\right)$ and $q=\left(q_{1}, q_{2}, \ldots, q_{n}\right)$ is defined by $p \wedge q=\left(p_{i} q_{j}-p_{j} q_{i}\right)_{i<j}$. The condition that the Gaussian curvature of $\Sigma_{a}$ is non-vanishing can be expressed as $\operatorname{det} \nabla^{2} a(\xi) \neq 0$ for all $\xi \neq 0$.

The third and fourth estimates (1.4) and (1.5) in Theorem 1.1 say that we can attain the critical order $s=1 / 2$ in the first and second estimates (1.2) and (1.3) under a certain structure condition. The operators appearing in (1.4) and (1.5) are related to the Laplace-Beltrami operator on $\Sigma_{a}$, but are of order zero in both $x$ and $\xi$. The precise geometric meaning of the structure (1.4) and (1.5) will be related to the Hamiltonian flow of the evolution governed by the defining function $a(\xi)$.

We also get the global version of the estimates, so that Theorem 1.1 follows by setting $\rho=1$ from the following:

Theorem 1.2. Assume conditions of Theorem 1.1. Then we have the uniform trace estimates

$$
\left\|f_{\mid \rho \Sigma_{a}}\right\|_{L^{2}\left(\rho \Sigma_{a} ; \rho^{n-1} d \omega\right)} \leq C\|f\|_{H^{s}\left(\mathbb{R}^{n}\right)} \quad(s>1 / 2),
$$

and

$$
\left\|f_{\mid \rho \Sigma_{a}}\right\|_{L^{2}\left(\rho \Sigma_{a} ; \rho^{n-1} d \omega\right)} \leq C \rho^{s-1 / 2}\|f\|_{\dot{H}^{s}\left(\mathbb{R}^{n}\right)} \quad(n / 2>s>1 / 2)
$$

for any $\rho>0$. Moreover, in the critical cases, we have

$$
\begin{aligned}
& \left\|\left(\frac{\nabla a(x)}{|\nabla a(x)|} \wedge \frac{D_{x}}{\left|D_{x}\right|}\right) f_{\mid \rho \Sigma_{a}}\right\|_{L^{2}\left(\rho \Sigma_{a} ; \rho^{n-1} d \omega\right)} \leq C\|f\|_{\dot{H}^{1 / 2}\left(\mathbb{R}^{n}\right)}, \\
& \left\|\left(\frac{x}{|x|} \wedge \frac{\nabla a^{*}\left(D_{x}\right)}{\left|\nabla a^{*}\left(D_{x}\right)\right|}\right) f_{\mid \rho \Sigma_{a}}\right\|_{L^{2}\left(\rho \Sigma_{a} ; \rho^{n-1} d \omega\right)} \leq C\|f\|_{\dot{H}^{1 / 2}\left(\mathbb{R}^{n}\right)},
\end{aligned}
$$


with constants independent of $\rho>0$.

We note that estimates (1.3), (1.4) and (1.5) are equivalent to estimates (1.7), (1.8) and (1.9) with the same constants $C$, respectively. Indeed, (1.7), (1.8) and (1.9) follow from estimates (1.3), (1.4) and (1.5) by just setting $f=f_{\rho}$, where the notation $g_{\rho}$ denotes the dilation $g_{\rho}(x)=g(\rho x)$. The converse is trivial.

Furthermore, we note that we get Theorem 1.2 with the critical case from the following generalised result:

Theorem 1.3. Let $a(\xi) \in C^{\infty}\left(\mathbb{R}^{n} \backslash 0\right)$ be real-valued and satisfy $a(\xi)>0$ and $a(\lambda \xi)=\lambda^{2} a(\xi)$ for $\lambda>0$ and $\xi \neq 0$. Assume that the Gaussian curvature of the set $\Sigma_{a}=\left\{\xi \in \mathbb{R}^{n} \backslash 0: a(\xi)=1\right\}$ never vanishes. Let a pseudo-differential operator $\sigma(X, D)$ have symbol $\sigma(x, \xi)$ which is smooth in $x \neq 0, \xi \neq 0$, and which is positively homogeneous of order $\beta$ in $x$ and of order $\alpha$ in $\xi$, i.e. $\sigma(\lambda x, \mu \xi)=\lambda^{\beta} \mu^{\alpha} \sigma(x, \xi)$ for all $x \neq 0, \xi \neq 0, \lambda>0, \mu>0$. Suppose also the structure condition

$$
\sigma(x, \lambda \nabla a(x))=0 \text { for all } x \neq 0 \text { and } \lambda \in \mathbb{R},
$$

or

$$
\sigma(-x, \lambda \nabla a(x))=0 \text { for all } x \neq 0 \text { and } \lambda \in \mathbb{R} .
$$

Then we have the estimate

$$
\left\|(\sigma(X, D) f)_{\mid \rho \Sigma_{a}}\right\|_{L^{2}\left(\rho \Sigma_{a} ; \rho^{n-1} d \omega\right)} \leq C \rho^{\beta}\|f\|_{\dot{H}^{1 / 2+\alpha}\left(\mathbb{R}^{n}\right)},
$$

with a constant $C$ independent of $\rho>0$. In particular, if $\alpha=\beta=0$, we obtain the uniform critical estimate

$$
\left\|(\sigma(X, D) f)_{\mid \rho \Sigma_{a}}\right\|_{L^{2}\left(\rho \Sigma_{a} ; \rho^{n-1} d \omega\right)} \leq C\|f\|_{\dot{H}^{1 / 2}\left(\mathbb{R}^{n}\right)} .
$$

Again we remark that estimates (1.11) and (1.12) are equivalent to themselves with $\rho=1$, with the same constant $C$, respectively. And we also remark that two types of conditions in (1.10) which have different sign coincide with each other if $a(\xi)$ is not only positively homogeneous but homogeneous.

In the next section we explore the geometric meaning of operators in (1.4) and (1.5) together with that of the structure condition (1.10) in more detail and show how these estimates can be deduced from the smoothing estimates for suitable evolution equations. For different results on smoothing estimates we refer to the first papers [12, 3, 8, 19, 22, and to the authors' paper [16] for the setting of evolution equations corresponding to this paper.

Finally, we remark that we can use the argument of this paper to also conclude some best constants. We give the statement corresponding to the case of the sphere, $a(\xi)=|\xi|^{2}$, in (1.3)

Theorem 1.4. Let $n / 2>s>1 / 2$. Then we have the estimate

$$
\left\|f_{\mid \mathbb{S}^{n-1}}\right\|_{L^{2}\left(\mathbb{S}^{n-1} ; d \omega\right)} \leq\left(2^{1-2 s} \frac{\Gamma(2 s-1) \Gamma\left(\frac{n}{2}-s\right)}{\Gamma(s)^{2} \Gamma\left(\frac{n}{2}-1+s\right)}\right)^{1 / 2}\|f\|_{\dot{H}^{s}\left(\mathbb{R}^{n}\right)},
$$

and the constant in estimate (1.13) can not be improved.

We can also conclude a more general result with a possibility to put different derivatives and weights. 
Theorem 1.5. Let $\sigma:(0, \infty) \rightarrow \mathbb{R}$ and $w:(0, \infty) \rightarrow \mathbb{R}$ be functions such that the following expression is finite:

$$
C_{1}=\left(\sup _{\substack{t>0 \\ k \in \mathbb{N}}}\left\{\frac{1}{\sigma(t)^{2}} \int_{0}^{\infty} J_{\nu(k)}(r t)^{2} \frac{r}{w(r)^{2}} d r\right\}\right)^{1 / 2}<\infty,
$$

where for $\lambda>-1 / 2$ the Bessel function $J_{\lambda}$ of order $\lambda$ is given by

$$
J_{\lambda}(t)=\frac{t^{\lambda}}{2^{\lambda} \Gamma(\lambda+1 / 2) \Gamma(1 / 2)} \int_{-1}^{1} e^{i t r}\left(1-r^{2}\right)^{\lambda-1 / 2} d r,
$$

and $\nu(k)=n / 2+k-1$. Then we have the estimate

$$
\left\|f_{\mid \rho \mathbb{S}^{n-1}}\right\|_{L^{2}\left(\rho \mathbb{S}^{n-1} ; \rho^{n-1} d \omega\right)} \leq C_{1} \sqrt{\rho} \sigma(\rho)\left\|w\left(\left|D_{x}\right|\right) f\right\|_{L^{2}\left(\mathbb{R}^{n}\right)},
$$

for all $\rho>0$, and the constant in estimate (1.16) can not be improved.

In particular, if we take $\sigma(t)=t^{s-1}$ and $w(t)=t^{s}$, the result is equivalent to Theorem 1.4. If we take $\sigma(t)=t^{-1 / 2}$ and $w(t)=\left(1+t^{2}\right)^{s / 2}(s>1 / 2)$, the result gives the best constant of estimate (1.6) in Theorem 1.2 in the case $a(\xi)=|\xi|^{2}$.

\section{Smoothing ESTimates AND TRACE THEOREMS}

Let us start with the result that was established by the authors in [15]. This concerns the critical case $(\alpha=1 / 2)$ of the Kato-Yajima's estimate

$$
\left\||x|^{\alpha-1}\left|D_{x}\right|^{\alpha} e^{i t a\left(D_{x}\right)} \varphi(x)\right\|_{L^{2}\left(\mathbb{R}_{t} \times \mathbb{R}_{x}^{n}\right)} \leq C\|\varphi\|_{L^{2}\left(\mathbb{R}_{x}^{n}\right)},
$$

which holds for $1-n / 2<\alpha<1 / 2$ (see [13] for $0 \leq \alpha<1 / 2$ and [20] for $1-n / 2<$ $\alpha<1 / 2$ in the case $a\left(D_{x}\right)=-\Delta_{x}$. For general $a\left(D_{x}\right)$, see [16, Theorem 5.2]). It was shown in [16] that this estimate for values of $\alpha$ close to $1 / 2$ implies the same estimate for smaller $\alpha$. Thus, the critical case of this estimate with $\alpha=1 / 2$ is important, especially since it can be applied to the well-posedness problems of the derivative nonlinear Schrödinger equations (see [17]). However, the estimate fails in the critical case $\alpha=1 / 2$ (see Watanabe [24], or the authors' paper [16] for more general negative results) and it is known that it is necessary to cut-off the radial derivatives for the estimate to hold in the critical case as well (see [21] or [16]). This can be done by replacing operator $\left|D_{x}\right|^{\alpha}$ by the Laplace-Beltrami operator on the sphere at the level $\alpha=1 / 2$. In fact, it turns out one can use any operator as long as its symbol vanishes on a certain set related to the symbol of the Laplace operator (the sphere is this case). To explain this precisely, let us formulate it for the equation

$$
\left\{\begin{aligned}
\left(i \partial_{t}+a\left(D_{x}\right)\right) u(t, x) & =0, \\
u(0, x) & =\varphi(x) \in L^{2}\left(\mathbb{R}_{x}^{n}\right),
\end{aligned}\right.
$$

where the real-valued function $a=a(\xi) \in C^{\infty}\left(\mathbb{R}^{n} \backslash 0\right)$ is elliptic and positively homogeneous of order two, that is, it satisfies $a(\xi)>0$ and $a(\lambda \xi)=\lambda^{2} a(\xi)$ for $\lambda>0$ and $\xi \neq 0$. We remark that under these condition we have the dispersiveness, namely, $\nabla a(\xi) \neq 0$ by the Euler's identity $a(\xi)=1 / 2 \nabla a(\xi) \cdot \xi$ and the ellipticity of $a(\xi)$. The case $a(\xi)=|\xi|^{2}$ corresponds to the usual Laplacian $a\left(D_{x}\right)=-\Delta_{x}$. 
Let us define $\{(x(t), y(t)): t \in \mathbb{R}\}$ to be the classical orbit, that is, the solution of the Hamilton-Jacobi ordinary differential equations

$$
\left\{\begin{array}{l}
\dot{x}(t)=\left(\nabla_{\xi} a\right)(\xi(t)), \quad \dot{\xi}(t)=0, \\
x(0)=0, \quad \xi(0)=\xi_{0},
\end{array}\right.
$$

and consider the set of the paths of all classical orbits

$$
\begin{aligned}
\Gamma_{a} & =\left\{(x(t), \xi(t)): t \in \mathbb{R}, \xi_{0} \in \mathbb{R}^{n} \backslash 0\right\} \\
& =\left\{(\lambda \nabla a(\xi), \xi): \xi \in \mathbb{R}^{n} \backslash 0, \lambda \in \mathbb{R}\right\} .
\end{aligned}
$$

Let a pseudo-differential operator $\sigma(X, D)$ have symbol $\sigma(x, \xi)$ which is smooth in $x \neq 0, \xi \neq 0$, and which is positively homogeneous of order $-1 / 2$ with respect to $x$, and of order $1 / 2$ with respect to $\xi$. Suppose also the structure condition

$$
\sigma(x, \xi)=0 \quad \text { if } \quad(x, \xi) \in \Gamma_{a} \quad \text { and } \quad x \neq 0 .
$$

Then it was shown in [15] that the solution $u=e^{i t a\left(D_{x}\right)} \varphi$ to (2.2) satisfies

$$
\left\|\sigma\left(X, D_{x}\right) e^{i t a\left(D_{x}\right)} \varphi(x)\right\|_{L^{2}\left(\mathbb{R}_{t} \times \mathbb{R}_{x}^{n}\right)} \leq C\|\varphi\|_{L^{2}\left(\mathbb{R}^{n}\right)}
$$

if $n \geq 2$ and the Gaussian curvature of the hypersurface

$$
\Sigma_{a}=\left\{\xi \in \mathbb{R}^{n} \backslash 0: a(\xi)=1\right\}
$$

never vanishes. We note that the set (2.3) and the assumption (2.4) are somehow related to Sommerfeld's radiation condition, see [17]. We also note that condition (1.10) in Theorem 1.3 means that $\check{\sigma}(x, \xi)$ or $\check{\sigma}(x,-\xi)$ satisfies the structure condition (2.4), where $\check{\sigma}(x, \xi)=\sigma(\xi, x)$ The typical example for such operator $\sigma\left(X, D_{x}\right)$ is given by the elements of

$$
\Omega_{1}=|x|^{-1 / 2}\left(\frac{x}{|x|} \wedge \frac{\nabla a\left(D_{x}\right)}{\left|\nabla a\left(D_{x}\right)\right|}\right)\left|D_{x}\right|^{1 / 2} .
$$

Another interesting example is the element of

$$
\Omega_{2}=|x|^{-1 / 2}\left(\frac{\nabla a^{*}(x)}{\left|\nabla a^{*}(x)\right|} \wedge \frac{D_{x}}{\left|D_{x}\right|}\right)\left|D_{x}\right|^{1 / 2},
$$

where $a^{*}(x)$ is the dual function of $a(\xi)$ which is positively homogeneous of order two and is characterised by the relation $a^{*}(\nabla a(\xi))=1$. We remark that the sum of the squares of all elements of $\Omega_{2}$ forms the main factor of the homogeneous extension of the Laplace-Beltrami operator on the dual hypersurface $\Sigma_{a}^{*}=\left\{\nabla a(\xi): \xi \in \Sigma_{a}\right\}$. The dual function $a^{*}(x)$ can be also determined by the relation $\Sigma_{a^{*}}=\Sigma_{a}^{*}$.

Let us say a few words regarding the dual hypersurfaces as it is shown in [15, Theorem 3.1]. Suppose $n \geq 2$. For the convenience of the formulation, in order to have the gradient of the function to be homogeneous of order zero, we may consider an extension of a function from its fixed level set as a positively homogeneous function of order one. Thus, let $p \in C^{\infty}\left(\mathbb{R}^{n} \backslash 0\right)$ be a positive and positively homogeneous function of order one. We assume that the Gaussian curvature of the hypersurface $\Sigma_{p}=\{\xi: p(\xi)=1\}$ never vanishes and we denote $\Sigma_{p}^{*}=\left\{\nabla p(\xi): \xi \in \Sigma_{p}\right\}$. Then there exists a unique positive and positively homogeneous function $p^{*} \in C^{\infty}\left(\mathbb{R}^{n} \backslash 0\right)$ of order one such that $\Sigma_{p}^{*}=\Sigma_{p^{*}}, \Sigma_{p^{*}}^{*}=\Sigma_{p}$, and the Gaussian curvature of the 
hypersurface $\Sigma_{p^{*}}$ never vanishes. Moreover, $\nabla p: \Sigma_{p} \rightarrow \Sigma_{p^{*}}$ is a $C^{\infty}$-diffeomorphism and $\nabla p^{*}: \Sigma_{p^{*}} \rightarrow \Sigma_{p}$ is its inverse.

The proof of Theorem 1.2 relies on the critical case of the limiting absorption principle which can be proved by reducing its statement to a model situation by the canonical transform method combined with weighted estimates for the transform operators. On the other hand, it can be reduced to a corresponding smoothing estimate for the Laplace operator with any critical operator, for example to the homogeneous extension of the Laplace-Beltrami operator on the sphere, recovering, in particular, the result of [21]. This particular case has been extended to include small perturbations by Barceló, Bennett and Ruiz [1]. For further details on these arguments we refer to authors' paper [15]. At the same time, the set $\Gamma_{a}$ corresponds to the Hamiltonian flow of $a\left(D_{x}\right)$, which is known to play a role in such problems also in a more general setting of manifolds. There, non-trapping conditions also enter (e.g. Doi [9, 10] in the case of Schrödinger operators on manifolds, using Egorov theorem, or Burq [6] and Burq, Gérard and Tzvetkov [7] in the case of Schrödinger boundary value problems, using propagation properties of Wigner measures), and such conditions can be also expressed in terms of properties of the set $\Gamma_{a}$. In our case this simply corresponds to the dispersiveness of $a\left(D_{x}\right)$. We also record the result for non-homogeneous weights, namely,

$$
\left\|\langle x\rangle^{-s}\left|D_{x}\right|^{1 / 2} e^{i t a\left(D_{x}\right)} \varphi(x)\right\|_{L^{2}\left(\mathbb{R}_{t} \times \mathbb{R}_{x}^{n}\right)} \leq C\|\varphi\|_{L^{2}\left(\mathbb{R}_{x}^{n}\right)}
$$

which holds for all $s>1 / 2$. For the Schrödinger case $a(\xi)=|\xi|^{2}$ and $n \geq 3$ this was shown by Ben-Artzi and Klainerman [4], and it was extended in [16], in particular, to any homogeneous of order 2 function $a(\xi)$ with $\nabla a(\xi) \neq 0$ for $\xi \neq 0, n \geq 1$. We will now prove Theorem 1.2 .

Proof of Theorem 1.2. First note that the formal adjoint $T^{*}: \mathcal{S}\left(\mathbb{R}_{t} \times \mathbb{R}_{x}^{n}\right) \rightarrow \mathcal{S}^{\prime}\left(\mathbb{R}_{x}^{n}\right)$ of the operator

$$
T=e^{i t a\left(D_{x}\right)}: \mathcal{S}\left(\mathbb{R}_{x}^{n}\right) \rightarrow \mathcal{S}^{\prime}\left(\mathbb{R}_{t} \times \mathbb{R}_{x}^{n}\right),
$$

the solution operator to equation (2.2), is expressed as

$$
T^{*}[v(t, x)]=\mathcal{F}_{\xi}^{-1}\left[\left(\mathcal{F}_{t, x} v\right)(a(\xi), \xi)\right] .
$$

Then, we claim that for any operator $A=A\left(X, D_{x}\right)$ acting on the variable $x$, the estimate

$$
\left\|A e^{i t a\left(D_{x}\right)} \varphi\right\|_{L^{2}\left(\mathbb{R}_{t} \times \mathbb{R}_{x}^{n}\right)} \leq C_{0}\|\varphi\|_{L^{2}\left(\mathbb{R}_{x}^{n}\right)}
$$

is equivalent to the estimate

$$
\begin{aligned}
\left\|\widehat{A}^{*} f_{\mid \rho \Sigma_{a}}\right\|_{L^{2}\left(\rho \Sigma_{a} ; 2 \rho^{n-1} d \omega /|\nabla a(\omega)|\right)} & \leq \frac{(2 \pi)^{n / 2}}{\sqrt{\pi}} C_{0} \sqrt{\rho}\|f\|_{L^{2}\left(\mathbb{R}^{n}\right)} \\
& =\frac{1}{\sqrt{\pi}} C_{0} \sqrt{\rho}\|\widehat{f}\|_{L^{2}\left(\mathbb{R}^{n}\right)} \quad \text { for all } \rho>0
\end{aligned}
$$


where $d \omega$ is the standard surface element of the hypersurface $\Sigma_{a}$ and $\rho \Sigma_{a}=\{\rho \omega$ : $\left.\omega \in \Sigma_{a}\right\}$. Indeed, by (2.10) and Plancherel's theorem, we have

$$
\begin{aligned}
\left\|T^{*} A^{*} v\right\|_{L^{2}\left(\mathbb{R}^{n}\right)}^{2} & =(2 \pi)^{-n}\left\|\left(\mathcal{F}_{t, x} A^{*} v\right)(a(\xi), \xi)\right\|_{L^{2}\left(\mathbb{R}_{\xi}^{n}\right)}^{2} \\
& =(2 \pi)^{-n} \int_{0}^{\infty}\left(\int_{\Sigma_{a}}\left|\left(\mathcal{F}_{t, x} A^{*} v\right)\left(\rho^{2}, \rho \omega\right)\right|^{2} \frac{2 \rho^{n-1} d \omega}{|\nabla a(\omega)|}\right) d \rho .
\end{aligned}
$$

Here we have used the change of variables $\xi \mapsto \rho \omega\left(\rho>0, \omega \in \Sigma_{a}\right)$. Then for $v(t, x)=g(t) f(x)$ we have

$$
\left\|T^{*} A^{*} v\right\|_{L^{2}\left(\mathbb{R}^{n}\right)}^{2}=(2 \pi)^{-n} \int_{0}^{\infty}\left|\widehat{g}\left(\rho^{2}\right) \sqrt{\rho}\right|^{2}\left(\int_{\Sigma_{a}}\left|\frac{1}{\sqrt{\rho}}\left(\widehat{A^{*} f}\right)(\rho \omega)\right|^{2} \frac{2 \rho^{n-1} d \omega}{|\nabla a(\omega)|}\right) d \rho .
$$

At the same time, by (2.11), we have

$$
\left\|T^{*} A^{*} v\right\|_{L^{2}\left(\mathbb{R}^{n}\right)}^{2} \leq C_{0}^{2}\|v\|_{L^{2}\left(\mathbb{R}_{t} \times \mathbb{R}_{x}^{n}\right)}^{2}=C_{0}^{2}\|g\|_{L^{2}(\mathbb{R})}^{2}\|f\|_{L^{2}\left(\mathbb{R}^{n}\right)}^{2} .
$$

Note that we have by Plancherel's theorem

$$
\|g\|_{L^{2}(\mathbb{R})}^{2}=\frac{1}{2 \pi}\|\widehat{g}\|_{L^{2}(\mathbb{R})}^{2}=\frac{1}{\pi} \int_{0}^{\infty}\left|\widehat{g}\left(\rho^{2}\right) \sqrt{\rho}\right|^{2} d \rho
$$

if supp $\widehat{g} \subset[0, \infty)$. Combining all these relations and taking arbitrary $g$, we obtain estimate (2.12).

Conversely, noting that $\left(\mathcal{F}_{t, x} A^{*} v\right)\left(\rho^{2}, \rho \omega\right)=\widehat{A}^{*} f_{\mid \rho \Sigma_{a}}$, where $f(\cdot)=\mathcal{F}_{t} v\left(\rho^{2}, \cdot\right)$, the right hand side of equality (2.13) can be estimated by using the first line of (2.12), and we actually have

$$
\begin{aligned}
\left\|T^{*} A^{*} v\right\|_{L^{2}\left(\mathbb{R}^{n}\right)}^{2} & \leq \frac{C_{0}^{2}}{\pi} \int_{0}^{\infty} \int_{\mathbb{R}^{n}}\left|\left(\mathcal{F}_{t} v\right)\left(\rho^{2}, x\right)\right|^{2} \rho d \rho d x \\
& =\frac{C_{0}^{2}}{2 \pi} \int_{0}^{\infty} \int_{\mathbb{R}^{n}}\left|\left(\mathcal{F}_{t} v\right)(\rho, x)\right|^{2} d \rho d x \\
& \leq \frac{C_{0}^{2}}{2 \pi}\left\|\mathcal{F}_{t} v\right\|_{L^{2}\left(\mathbb{R}_{t} \times \mathbb{R}_{x}^{n}\right)}^{2}=C_{0}^{2}\|v\|_{L^{2}\left(\mathbb{R}_{t} \times \mathbb{R}_{x}^{n}\right)}^{2}
\end{aligned}
$$

for any $v(t, x) \in L^{2}\left(\mathbb{R}_{t} \times \mathbb{R}_{x}^{n}\right)$, which implies estimate (2.11). We remark that (2.12) implies a corresponding version of the limiting absorption principle for $a\left(D_{x}\right)$, which, in turn, means that $A$ is $a\left(D_{x}\right)$-supersmooth, see e.g. Kato [11] or Ben-Artzi and Devinatz [2]. We will not discuss further details of this here as we do not need it in this paper.

We have already reviewed examples of operators $A$ which satisfy smoothing estimate (2.11), hence the Fourier restriction estimate (2.12). For example, using (2.9) and (2.1), we can take

$$
\begin{aligned}
& A_{1}=\langle x\rangle^{-s}\left|D_{x}\right|^{1 / 2} \quad(s>1 / 2), \\
& A_{2}=|x|^{\alpha-1}\left|D_{x}\right|^{\alpha} \quad(1-n / 2<\alpha<1 / 2) .
\end{aligned}
$$

We can also take $A=\sigma\left(X, D_{x}\right)$ which appeared in estimate (2.5), especially the elements of the operators $\Omega_{1}$ or $\Omega_{2}$ defined by (2.7) or (2.8), but in this case we also need the non-degenerate Gaussian curvature condition on the hypersurface $\Sigma_{a}$ 
defined by (2.6), which is equivalent to $\operatorname{det} \nabla^{2} a(\xi) \neq 0(\xi \neq 0)$ (see Miyachi [14], for example). Their formal adjoints are given by

$$
\begin{aligned}
& A_{1}^{*}=\left|D_{x}\right|^{1 / 2}\langle x\rangle^{-s} \quad(s>1 / 2), \\
& A_{2}^{*}=\left|D_{x}\right|^{1-s}|x|^{-s} \quad(n / 2>s>1 / 2)
\end{aligned}
$$

(here we take $s=1-\alpha$ for $A_{2}$ ), and also

$$
\begin{aligned}
& \Omega_{1}^{*}=\left|D_{x}\right|^{1 / 2}\left(\frac{\nabla a\left(D_{x}\right)}{\left|\nabla a\left(D_{x}\right)\right|} \wedge \frac{x}{|x|}\right)|x|^{-1 / 2}, \\
& \Omega_{2}^{*}=\left|D_{x}\right|^{1 / 2}\left(\frac{D_{x}}{\left|D_{x}\right|} \wedge \frac{\nabla a^{*}(x)}{\left|\nabla a^{*}(x)\right|}\right)|x|^{-1 / 2} .
\end{aligned}
$$

Note that we have $|\nabla a(\xi)| \geq C>0$ on $\Sigma_{a}$ since $\nabla a(\xi) \neq 0(\xi \neq 0)$ in our case. From the construction, we have the same property for $a^{*}$, as well. We also note that $\|f\|_{L_{s}^{2}\left(\mathbb{R}^{n}\right)}=(2 \pi)^{-n / 2}\|\widehat{f}\|_{H^{s}\left(\mathbb{R}^{n}\right)}$ and $\|f\|_{\dot{L}_{s}^{2}\left(\mathbb{R}^{n}\right)}=(2 \pi)^{-n / 2}\|\widehat{f}\|_{\dot{H}^{s}\left(\mathbb{R}^{n}\right)}$, for weighted $L^{2}$-spaces $L_{s}^{2}\left(\mathbb{R}^{n}\right)$ and $\dot{L}_{s}^{2}\left(\mathbb{R}^{n}\right)$ defined by the norms $\|f\|_{L_{s}^{2}\left(\mathbb{R}^{n}\right)}=\left\|\langle x\rangle^{s} f\right\|_{L^{2}\left(\mathbb{R}^{n}\right)}$ and $\|f\|_{\dot{L}_{s}^{2}\left(\mathbb{R}^{n}\right)}=\left\||x|^{s} f\right\|_{L^{2}\left(\mathbb{R}^{n}\right)}$, respectively.

Now, first, using (2.9), we get (2.11) with operator $A_{1}$ in (2.14), from which, on account of (2.12), we can conclude the following trace result:

$$
\left\|f_{\mid \rho \Sigma_{a}}\right\|_{L^{2}\left(\rho \Sigma_{a} ; \rho^{n-1} d \omega\right)} \leq C\|f\|_{H^{s}\left(\mathbb{R}^{n}\right)} \quad(s>1 / 2) .
$$

Here we remark that $\nabla a \neq 0$ because of Euler's identity $a(\xi)=(1 / 2) \xi \cdot \nabla a(\xi)>0$. If we use $A_{2}$ in (2.14) instead, and estimate (2.1), we get

$$
\left\|f_{\mid \rho \Sigma_{a}}\right\|_{L^{2}\left(\rho \Sigma_{a} ; \rho^{n-1} d \omega\right)} \leq C \rho^{s-1 / 2}\|f\|_{\dot{H}^{s}\left(\mathbb{R}^{n}\right)} \quad(n / 2>s>1 / 2) .
$$

In the critical cases, using estimate (2.5) with operators $\Omega_{1}$ or $\Omega_{2}$ defined by (2.7) or (2.8), and formulae for their adjoints, we obtain

$$
\begin{aligned}
& \left\|\left(\frac{\nabla a(x)}{|\nabla a(x)|} \wedge \frac{D_{x}}{\left|D_{x}\right|}\right) f_{\mid \rho \Sigma_{a}}\right\|_{L^{2}\left(\rho \Sigma_{a} ; \rho^{n-1} d \omega\right)} \leq C\|f\|_{\dot{H}^{1 / 2}\left(\mathbb{R}^{n}\right)}, \\
& \left\|\left(\frac{x}{|x|} \wedge \frac{\nabla a^{*}\left(D_{x}\right)}{\left|\nabla a^{*}\left(D_{x}\right)\right|}\right) f_{\mid \rho \Sigma_{a}}\right\|_{L^{2}\left(\rho \Sigma_{a} ; \rho^{n-1} d \omega\right)} \leq C\|f\|_{\dot{H}^{1 / 2}\left(\mathbb{R}^{n}\right)} .
\end{aligned}
$$

This completes the proof.

We now show that, in fact, the same argument yields a more general result.

Proof of Theorem 1.3. Let us assume the first part of condition (1.10). As for the case when we assume the second part, the result is obtained straightforwardly from the relation

$$
(\sigma(X, D) f)(x)=\overline{(\bar{\sigma}(-X, D)(\bar{f}(-x)))(-x)} .
$$

Let us define $A(X, D)=|X|^{-1 / 2-\alpha} \check{\sigma}(X, D)\left|D_{x}\right|^{1 / 2-\beta}$, where $\check{\sigma}(x, \xi)=\sigma(\xi, x)$ Then its symbol $A(x, \xi)=|x|^{-1 / 2-\alpha} \check{\sigma}(x, \xi)|\xi|^{1 / 2-\beta}$ satisfies $A(x, \xi)=0$ if $(x, \xi) \in \Gamma_{a}$ and $x \neq 0, \xi \neq 0$. Moreover, the symbol $A(x, \xi)$ is homogeneous of order $-1 / 2$ in $x$ and of order $1 / 2$ in $\xi$. Consequently, the operator $A(X, D)$ satisfies (2.4) and (2.5). The 
argument in the proof of Theorem 1.2 yields the estimate (2.12). Taking $g$ so that $\mathcal{F}^{-1} \bar{g}(x)=|x|^{-1 / 2-\alpha} f(x)$, we obtain

$$
\rho^{1 / 2-\beta}\left\|\mathcal{F}\left(\check{\sigma}(X, D)^{*} \mathcal{F}^{-1} \bar{g}\right)_{\mid \rho \Sigma_{a}}\right\|_{L^{2}\left(\rho \Sigma_{a} ; \rho^{n-1} d \omega /|\nabla a|\right)} \leq C \sqrt{\rho}\|g\|_{\dot{H}^{1 / 2+\alpha}\left(\mathbb{R}_{x}^{n}\right)} .
$$

We can now readily check that

$$
\mathcal{F}\left(\check{\sigma}(X, D)^{*} \mathcal{F}^{-1} \bar{g}\right)(x)=\overline{(\sigma(X, D) g)(x)} .
$$

Indeed, writing formally

$$
\left(\check{\sigma}(X, D)^{*} h\right)(x)=\mathcal{F}_{\xi}^{-1}\left(\int_{\mathbb{R}^{n}} e^{-i y \cdot \xi} \overline{\check{\sigma}(y, \xi)} h(y) d y\right)(x),
$$

we get

$$
\mathcal{F}\left(\check{\sigma}(X, D)^{*} \mathcal{F}^{-1} \bar{g}\right)(\eta)=\int_{\mathbb{R}^{n}} e^{-i y \cdot \eta} \overline{\sigma(\eta, y)} \mathcal{F}^{-1} \bar{g}(y) d y,
$$

yielding (2.16). Consequently, from estimate (2.15) and equality (2.16) we obtain (1.11).

On account of the argument in the proof of Theorem[1.2, it is apparent that the best constants of trace theorems for the sphere $\mathbb{S}^{n-1}$ are obtained from those of smoothing estimates for Schrödinger case $a(\xi)=|\xi|^{2}$.

Proof of Theorem 1.4. The best constant $C_{0}$ of estimate (2.11) with $A=A_{2}$ in (2.14) is

$$
\begin{aligned}
C_{0} & =\left(\pi 2^{2 \alpha-1} \frac{\Gamma(1-2 \alpha) \Gamma\left(\frac{n}{2}+\alpha-1\right)}{\Gamma(1-\alpha)^{2} \Gamma\left(\frac{n}{2}-\alpha\right)}\right)^{1 / 2} \\
& =\left(\pi 2^{1-2 s} \frac{\Gamma(2 s-1) \Gamma\left(\frac{n}{2}-s\right)}{\Gamma(s)^{2} \Gamma\left(\frac{n}{2}-1+s\right)}\right)^{1 / 2},
\end{aligned}
$$

where $s=1-\alpha$ (see [5]). We remark that this constant with $\alpha=0$, that is, $C_{0}=\sqrt{\pi /(n-2)}$ was given by an earlier work of Simon [18]. Then by the argument of the proof of Theorem [1.2, we have estimate (2.12) with the same $C_{0}$ as the best one. Since it is equivalent to itself with $\rho=1$, we have the conclusion.

Proof of Theorem 1.5. We recall that in general that if $n \geq 2$ and $g$ is injective and differentiable on $(0, \infty)$, the best constant $C_{0}$ in the inequality

$$
\left\|w(|x|)^{-1} \sigma\left(\left|D_{x}\right|\right)^{-1} e^{i t g\left(\left|D_{x}\right|\right)} \varphi(x)\right\|_{L^{2}\left(\mathbb{R}_{t} \times \mathbb{R}_{x}^{n}\right)} \leq C_{0}\|\varphi\|_{L^{2}\left(\mathbb{R}_{x}^{n}\right)}
$$

is given by

$$
C_{0}=\left(2 \pi \sup _{\substack{\rho>0 \\ k \in \mathbb{N}}}\left\{\frac{\rho}{\sigma(\rho)^{2} g^{\prime}(\rho)} \int_{0}^{\infty} J_{\nu(k)}(r \rho)^{2} \frac{r}{w(r)^{2}} d r\right\}\right)^{1 / 2},
$$

where for $\lambda>-1 / 2$ the Bessel function $J_{\lambda}$ of order $\lambda$ is given by (1.15) (see also [5]). This expression for the best constants was obtained by Walther [23, and it can be used to analyse estimates for radially symmetric equations by carefully looking at the asymptotic behaviour of Bessel functions and subsequent integrals. We now 
take $g(\rho)=\rho^{2}$ and $A=w(|x|)^{-1} \sigma\left(\left|D_{x}\right|\right)^{-1}$. Estimate (2.17) now implies (2.12) with $A^{*}=\sigma\left(\left|D_{x}\right|\right)^{-1} w(|x|)^{-1}$. Consequently, we get

$$
\left\|\sigma(|x|)^{-1} w(|D|)^{-1} f_{\mid \rho \mathbb{S}^{n-1}}\right\|_{L^{2}\left(\rho \mathbb{S}^{n-1} ; \rho^{n-1} d \omega\right)} \leq \frac{1}{\sqrt{\pi}} C_{0} \sqrt{\rho}\|f\|_{L^{2}\left(\mathbb{R}^{n}\right)},
$$

which implies (1.16).

\section{REFERENCES}

[1] J.A. Barceló, J.M. Bennett and A. Ruiz, Spherical perturbations of Schrödinger equations, J. Fourier Anal. Appl. 12 (2006), 269-290.

[2] M. Ben-Artzi and A. Devinatz, The limiting absorption principle for partial differential operators, Mem. Amer. Math. Soc. 66 (1987).

[3] M. Ben-Artzi and A. Devinatz, Local smoothing and convergence properties of Schrödinger type equations, J. Funct. Anal. 101 (1991), 231-254.

[4] M. Ben-Artzi and S. Klainerman, Decay and regularity for the Schrödinger equation, J. Analyse Math. 58 (1992), 25-37.

[5] N. Bez and M. Sugimoto, Optimal constants and extremisers for some smoothing estimates, arXiv:1206.5110

[6] N. Burq, Smoothing effect for Schrödinger boundary value problems, Duke Math. J. 123 (2004), 403-427.

[7] N. Burq, P. Gérard and N. Tzvetkov, On nonlinear Schrödinger equations in exterior domains, Ann. Inst. H. Poincare Anal. Non Lineaire 21 (2004), 295-318.

[8] P. Constantin and J. C. Saut, Local smoothing properties of dispersive equations, J. Amer. Math. Soc. 1 (1988), 413-439.

[9] S. Doi, Smoothing effects of Schrödinger evolution groups on Riemannian manifolds, Duke Math. J. 82 (1996), 679-706.

[10] S. Doi, Smoothing effects for Schrodinger evolution equation and global behavior of geodesic flow, Math. Ann. 318 (2000), 355-389.

[11] T. Kato, Wave operators and similarity for some non-selfadjoint operators, Math. Ann. 162 (1965/1966), 258-279.

[12] T. Kato, On the Cauchy problem for the (generalized) Korteweg-de Vries equation, Studies in applied mathematics, 93-128, Adv. Math. Suppl. Stud., 8, Academic Press, New York, 1983.

[13] T. Kato and K. Yajima, Some examples of smooth operators and the associated smoothing effect, Rev. Math. Phys. 1 (1989), 481-496.

[14] A. Miyachi, On some estimates for the wave equation in $L^{p}$ and $H^{p}$, J. Fac. Sci. Univ. Tokyo Sect. IA Math. 27 (1980), 331-354.

[15] M. Ruzhansky and M. Sugimoto, A smoothing property of Schrödinger equations in the critical case, Math. Ann. 335 (2006), 645-673.

[16] M. Ruzhansky and M. Sugimoto, Smoothing estimates of evolution equations via canonical transforms and comparison, Proc. London Math. Soc. (2012), doi: 10.1112/plms/pds006.

[17] M. Ruzhansky and M. Sugimoto, Structural resolvent estimates and derivative nonlinear Schrödinger equations, Comm. Math. Phys. (2012), doi: 10.1007/s00220-012-1524-x.

[18] B. Simon, Best constants in some operator smoothness estimates, J. Funct. Anal. 107 (1992), 66-71.

[19] P. Sjölin, Regularity of solutions to the Schrödinger equation, Duke Math. J. 55 (1987), 699-715.

[20] M. Sugimoto, Global smoothing properties of generalized Schrödinger equations, J. Anal. Math. 76 (1998), 191-204.

[21] M. Sugimoto, A Smoothing property of Schrödinger equations along the sphere, J. Anal. Math. 89 (2003), 15-30.

[22] L. Vega, Schrödinger equations: Pointwise convergence to the initial data, Proc. Amer. Math. Soc. 102 (1988), 874-878.

[23] B. G. Walther, Regularity, decay, and best constants for dispersive equations, J. Funct. Anal. 189 (2002), 325-335. 
[24] K. Watanabe, Smooth perturbations of the selfadjoint operator $|\Delta|^{\alpha / 2}$, Tokyo J. Math. 14 (1991), 239-250.

Michael Ruzhansky:

Department of Mathematics

IMPERIAL COLLEGE LONDON

180 QueEn's Gate, London SW7 2AZ, UK

E-mail address m.ruzhansky@imperial.ac.uk

Mitsuru Sugimoto:

Graduate School of Mathematics

NAGOYA UNIVERSITY

Furocho, Chikusa-Ku, NAGOYa 464-8602, JAPAN

E-mail address sugimoto@math.nagoya-u.ac.jp 